\title{
13. STELLAR CHEMICAL EVOLUTION
}




\title{
STELLAR CHEMICAL EVOLUTION
}

\author{
JOHN E. NORRIS \\ Mount Stromlo \& Siding Spring Observatories, \\ The Australian National University, \\ Private Bag, Woden P.O., ACT 2611, Australia \\ email: jen@mso.anu.edu.au
}

\section{Introduction}

One of the major achievements of astrophysics has been the demonstration that most of the chemical elements have been synthesized in stars: nucleosynthesis calculations of homogeneous and inhomogeneous big bang cosmologies show that, in comparison with the most metal-poor stars currently known, essentially no elements heavier than B existed at the era of decoupling (see e.g. Wagoner, Fowler, \& Hoyle 1967; Kajino, Mathews, \& Fuller 1990). Following the pioneering work on stellar nucleosynthesis by Hoyle (1946), the basic precepts and the role of stars was set down in the classic papers of Burbidge et al. (1957) and Cameron (1957), and the ensuing decades have produced a vast body of theoretical and observational effort to more fully understand the details of the process.

Extensive reviews of the subject exist in the literature. Some, which the reader may find useful, are those of Arnett (1996), Trimble (1975), Truran (1984), and Wheeler, Sneden, \& Truran (1989).

The nature of the first heavy-element-producing objects remains the subject of conjecture. Authors such as Couchman \& Rees (1986) argue that the first zero-heavy-element-abundance stars formed as the result of cooling via the hydrogen molecule in clusters of mass $\sim 10^{5} \mathrm{M}_{\odot}$. While the mass function of this first stellar generation remains elusive its evolution will have two important effects. The first supernovae would halt any ongoing collapse of material to form further stars, and more important for the present discussion, the ejecta from these objects would chemically enhance the remaining gaseous material: Couchman \& Rees advocate possible enrichment up to Population II values (i.e. $\mathrm{Z} / \mathrm{Z}_{\odot} \sim 0.01$ ).

It is interesting then to note that the low column density Ly $\alpha$ clouds inferred from the spectra of quasars appear to have abundances near this 
value (Cowie et al. 1995), and that at redshifts of $\sim 3$ a similar result is found for the damped Ly $\alpha$ systems (Pettini et al. 1994). In the Galaxy one can look at things a little more closely: for the halo globular clusters $\langle[\mathrm{Fe} / \mathrm{H}]\rangle=-1.6$, with the lowest values found at $\sim-2.3$ (Zinn 1985). The halo stars yield more detail because of their greater number: to first approximation their abundance distribution is similar to that of the simple closed-box model of chemical enrichment, with normal stars of lowest abundance having $[\mathrm{Fe} / \mathrm{H}]=-4.0$ (Ryan \& Norris 1991; McWilliam et al. 1995; Ryan, Norris, \& Beers 1996). At the other abundance extreme, the most metal-rich material in the Galaxy for which high quality abundances exist occurs in the bulge, with $[\mathrm{Fe} / \mathrm{H}] \sim 0.5$ (McWilliam \& Rich 1994).

A large body of observational material exists to test and guide theoretical studies. The aim here is to present a comparison between the results of the two approaches. Following a brief section on each, comparison will be made between observed and predicted relative abundances, $[\mathrm{X} / \mathrm{Fe}]$, as a function of $[\mathrm{Fe} / \mathrm{H}]$. Broad general agreement is found, but important exceptions exist, and an attempt will be made to highlight these differences, with conjectures as to how the situation might be improved.

\section{Theory}

The production of the elements during stellar evolution has been the subject of intense theoretical study during the past five decades, and the reader is referred to Arnett (1996), and references therein, for a thorough discussion of the topic. Suffice it here to say that following relatively quiescent evolution stars possess a series of concentric regions containing the ashes of various phases of nuclear burning. Major uncertainties remain concerning the manner in which material is undoubtedly mixed within stellar interiors over and above the predictions of standard theories and in which it is lost by winds during the relatively quiescent stages. The expulsion and burning of material in the final explosive phases of the more massive stars remain the subject of great activity.

The picture has emerged of a large fraction of the elements having been produced in massive stars, in the range $10<\mathrm{M} / \mathrm{M}_{\odot}<30$. In particular, these relatively short-lived objects have produced some $\mathrm{C}$, most of the $\mathrm{O}$ and elements in the range $\mathrm{Ne}-\mathrm{Ca}$, the iron-peak elements, and some of the heavier neutron-capture elements via the r-process. Large uncertainties remain, however, in the modelling. The recent supernova simulations, for example, of Woosley \& Weaver (1995) show a strong sensitivity to the assumed energy release: for $\mathrm{M}=25 \mathrm{M}_{\odot}, \mathrm{Z}=0$ higher energy explosions result in iron-peak element production with a neutron star remnant, while less energetic ones produce no iron and result in a black hole. In the latter 
case more $\mathrm{C}$ is produced than $\mathrm{O}$, in contradistinction to what normally happens. The complexity of the situation suggests that advances will only be made by a strong interaction between theory and observation, as has been emphasized by Arnett (1996).

Less massive objects, in the range $1<\mathrm{M} / \mathrm{M}_{\odot}<10$, are believed to be responsible for much of the $\mathrm{C}$ and $\mathrm{N}$, and for most of the heavy neutroncapture elements via the s-process. Uncertainties remain: the $\mathrm{C} / \mathrm{N}$ ratio depends critically of the treatment of convection (see Renzini \& Voli 1981), and the neutron source for the production of the heaviest elements, once believed to be the ${ }^{22} \mathrm{Ne}(\alpha, \mathrm{n})^{25} \mathrm{Mg}$ reaction in stars of mass 4-8 $\mathrm{M}_{\odot}$ (Iben \& Truran 1978) is now thought, following the revision of nuclear cross sections, to be due to ${ }^{13} \mathrm{C}(\alpha, \mathrm{n}){ }^{16} \mathrm{O}$ in stars of lower mass (Busso et al. 1988).

Type Ia supernovae, believed to involve the deflagration of accreting white dwarfs in binary systems, are invoked to produce large amounts of Fe at later times (see e.g. Nomoto et al. 1984).

In is important to note, however, that in spite of the large uncertainties a judicious choice of the free parameters can lead to a good reproduction of observed abundance patterns. Timmes, Woosley, \& Weaver (1995; their Figure 5), for example, present theoretical results for solar neighborhood material which reproduce the observed relative abundances for elements $\mathrm{H}-\mathrm{Co}$ to within a factor 2 . Given that the absolute abundances range over a factor of $8 \mathrm{dex}$, this is no mean achievement.

\section{Observation}

Improvements in the theory of stellar atmospheres and in detector technology in the past two decades have resulted in observed abundances of very high internal precision for relatively large numbers of stars. Two examples of this are the works of Edvardsson et al. (1993) and McWilliam et al. (1995), where internal precisions of $\sim 0.05-0.15$ dex are claimed, and indicated by the internal consistency of the material presented.

Here too, however, the treatment of convection in the models is the subject of concern, as had been emphasized by Kurucz in this volume. At its simplest this may lead to small systematic differences when using model atmospheres from different sources (see e.g. Ryan et al. [1996] for an example of differences of $\sim 0.10 \mathrm{dex}$ ), while basic inadequacies in the current treatments may compromise all current results. To the present author, at least, the internal consistency of abundances reported by Boesgaard \& Friel (1990) for the dwarfs in the Hyades and Pleiades, and in the case of the former their similarity to values given for its giants by Lambert \& Ries (1981) suggest that the current techniques lead to reasonable results, at least at the 0.1 dex level. 


\section{Comparison between Observation and Theory}

A detailed comparison for all elements lies outside the limitations of the present discussion. In what follows attention will be restricted to CNO, the $\alpha$ elements $\mathrm{Mg}, \mathrm{Si}, \mathrm{Ca}$, and $\mathrm{Ti}$, the odd-numbered light elements $\mathrm{Na}$ and $\mathrm{Al}$, the iron-peak elements, and the heavy neutron-capture elements, which are most amenable to observation and representative of the problem.

\subsection{CARBON, NITROGEN, AND OXYGEN}

The agreement for the CNO group as a function of metallicity is a mixed bag. A useful comparison of theory with observation is presented by Timmes et al. (1995; Figures 11, 13, and 14). In the view of the author the agreement is good for $\mathrm{O}$, needs improvement for $\mathrm{C}$, and is very poor for $\mathrm{N}$.

For $\mathrm{O}$ the more recent data of King (1994) together with the results of Timmes et al. show excellent agreement with the prediction of $[\mathrm{O} / \mathrm{Fe}] \sim 0.4$ for $[\mathrm{Fe} / \mathrm{H}]<-1.0$ from Type II supernovae. The data of Edvardsson et al. (1993) show a well-defined decrease in $[\mathrm{O} / \mathrm{Fe}]$ as $[\mathrm{Fe} / \mathrm{H}]$ increases from -1.0 to solar, as expected at later times following enrichment by the O-poor, Fe-rich ejecta of deflagration supernovae.

The situation for $\mathrm{C}$ is not so clear. Timmes et al. predict that $[\mathrm{C} / \mathrm{Fe}] \sim$ 0.0 for $-3.0<[\mathrm{Fe} / \mathrm{H}]<0.0$. In comparison Wheeler et al. (1989) noted that over the range $-2.5<[\mathrm{Fe} / \mathrm{H}]<-1.5[\mathrm{C} / \mathrm{Fe}]$ appeared to rise slightly towards lower abundance, and the results of McWilliam et al. (1995) and Norris, Ryan, \& Beers (1997) are suggestive of the effect continuing at even lower abundance. (Note that internal mixing effects such as the $\mathrm{C}$ depletions seen in globular cluster stars act in the opposite direction.) If $\mathrm{C}$ overabundances at lowest abundances are confirmed by future observation, they will provide a challenge to the model builders to determine whether the problem lies with the theoretical yields of $\mathrm{C}$ in the $10-30 \mathrm{M}_{\odot}$ objects or a more exotic source must be sought for this element.

The comparison of Timmes et al. for $\mathrm{N}$ shows quite clearly that, observationally, it is produced as a primary element in the most metal-poor stars, with the data suggesting $[\mathrm{N} / \mathrm{Fe}] \sim 0.0$ for $-3.0<[\mathrm{Fe} / \mathrm{H}]<0.0$, while the models quite starkly insist that it is secondary. This provides perhaps the clearest demonstration that at high mass, as has been evident for some time for low-mass objects, existing theory is quite unable to mix $\mathrm{N}$ produced in stellar interiors into more exterior regions. Several mechanisms suggest themselves to overcome the problem. Timmes et al. produce primary $\mathrm{N}$ by increasing convective overshoot in their models; Langer, in this volume, suggests that rotation may lead to greater production of $\mathrm{N}$; and a more exotic solution might be the $500 \mathrm{M}_{\odot}, \mathrm{Z}=0$ hypernovae of Woosley \& Weaver (1982), which produce copious amounts of $\mathrm{N}$. 


\subsection{THE $\alpha$ ELEMENTS}

A comparison between observation and theory for the four $\alpha$ elements $\mathrm{Mg}$, $\mathrm{Si}, \mathrm{Ca}$, and $\mathrm{Ti}$ is shown in Figure 1, which has been taken from Ryan et al. (1996). On the left are the observations, where different symbols show the results of different workers. On the right the data are presented again as small symbols. Here the thin lines are robust estimators of the abundance trends, with the central one being the running mid-mean. The theoretical results for the Galactic enrichment model of Timmes et al. ${ }^{1}$ (1995) are shown by thick dashed lines, where the lower refers to their basic calculations and the upper to their preferred result when the Fe from the model supernovae is arbitrarily reduced by a factor 2 . With the possible exception of $\mathrm{Ti}$ there is good agreement between observation and theory.

The data of Edvardsson et al. (1993) for these elements at $[\mathrm{Fe} / \mathrm{H}]>-$ 1.0 show very clearly the transition from the enhanced Population II values to solar as the result of Type Ia supernova production of Fe-enriched, $\alpha$ element-poor material at later times. Somewhat puzzling, however, are the results of McWilliam \& Rich (1994) for giants in the Galactic bulge. Their stars have $[\mathrm{Fe} / \mathrm{H}]>-1.0$, but different values of $[\mathrm{Mg} / \mathrm{Fe}]$ and $[\mathrm{Ti} / \mathrm{Fe}]$, on the one hand, and $[\mathrm{Si} / \mathrm{Fe}]$ and $[\mathrm{Ca} / \mathrm{Fe}]$, on the other. As noted by them, this is difficult to understand from a theoretical point of view. More work, both theoretical and observational (since the result is based on a relatively small number of faint objects), is needed to resolve this important issue.

\section{3. $\mathrm{AL}, \mathrm{NA}$}

The odd-atomic numbered elements $\mathrm{Al}$ and $\mathrm{Na}$ are discussed here to highlight a couple of problems. Theory predicts that they will be underproduced relative to neighboring even-numbered elements in low abundance environments, and this appears to be borne out by observation, at least in principle if not in degree. In particular Ryan et al. (1996) show that for $[\mathrm{Fe} / \mathrm{H}]<-2.0$ $[\mathrm{Al} / \mathrm{Fe}] \sim-0.8$, while Timmes et al. (1995) predict a value in the range -0.4 to 0.0. As Ryan et al. note, however, their abundances were determined assuming LTE, while the preliminary work of Baumueller and Gehren shows that large non-LTE effects, sufficient to explain the observed difference, become important at these low abundances.

As discussed by $\mathrm{Da}$ Costa in this volume, $\mathrm{Al}$ and $\mathrm{Na}$, together with $\mathrm{Mg}$ (see also the paper by Shetrone herein), show very peculiar abundances in globular clusters, not predicted by standard stellar evolution calculations,

\footnotetext{
${ }^{1}$ As emphasized by Gibson (1997) different workers sometimes produce different yields for the same input model parameters. While comparison is given here with Timmes et al., one should bear in mind that comparison with other workers might give somewhat different results.
} 

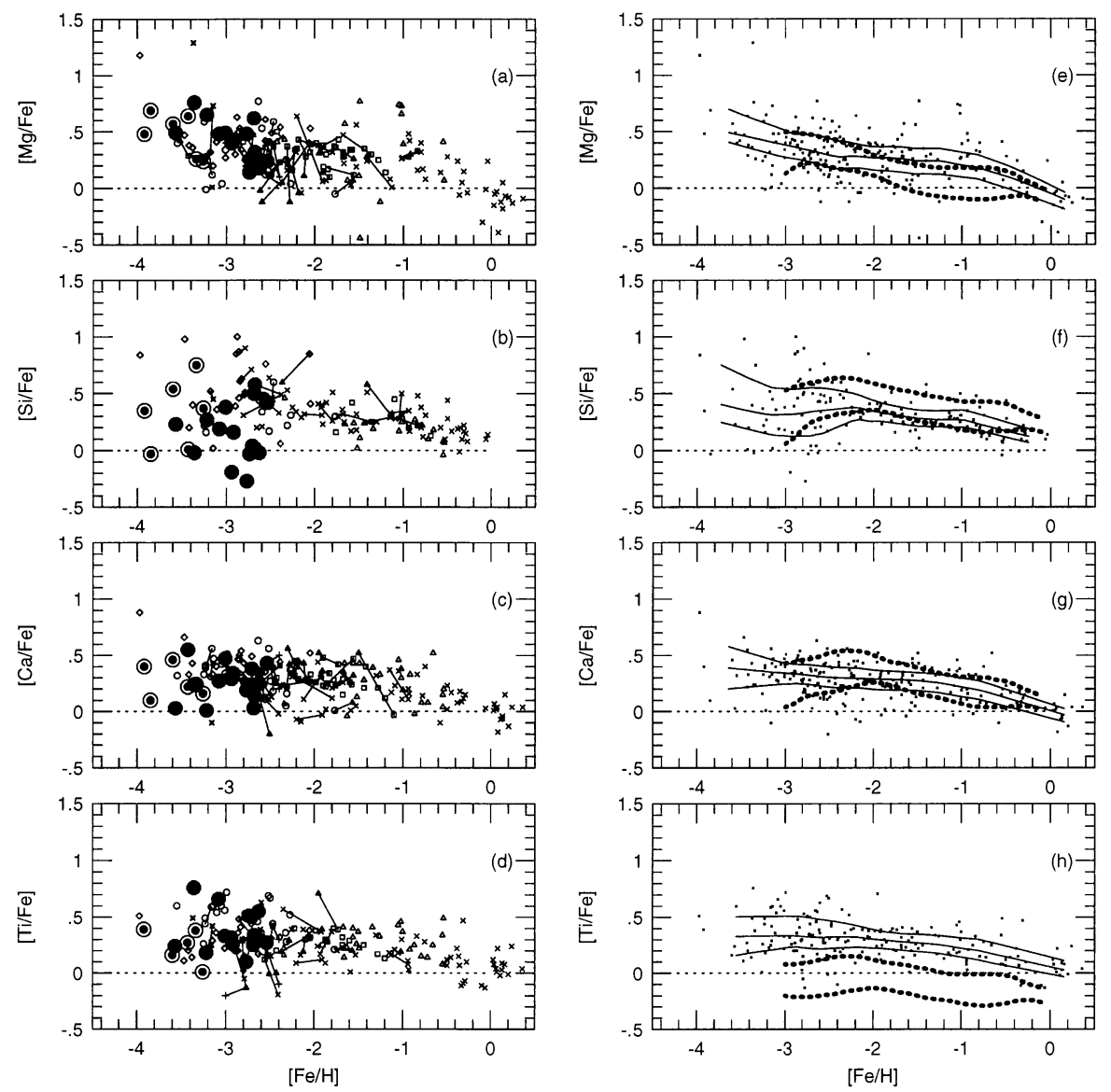

Figure 1. Relative abundances of the $\alpha$ elements as a function of $[\mathrm{Fe} / \mathrm{H}]$ from Ryan et al. (1996). On the left different symbols present results from different workers, while on the right the data are again presented as small symbols. The thin lines represent an averaging of the data, while the dashed lines come from the Galactic enrichment model of Timmes et al. (1995). See text for discussion.

but thought nevertheless to originate from $(p, \gamma)$ reactions in or near the hydrogen burning shells of clusters subgiants. The report by Briley et al. (1996), however, of $\mathrm{Na}$ abundance variations at the main sequence turnoff of 47 Tuc offers a challenge to this hypothesis and, unless the variations existed in the cluster at the formation of the Na-enhanced stars, suggests an important confrontation between theory and observation.

\subsection{THE IRON-PEAK ELEMENTS}

Figure 2 presents a comparison between observation and theory for the abundances of the iron-peak elements as a function of $[\mathrm{Fe} / \mathrm{H}]$. This demon- 

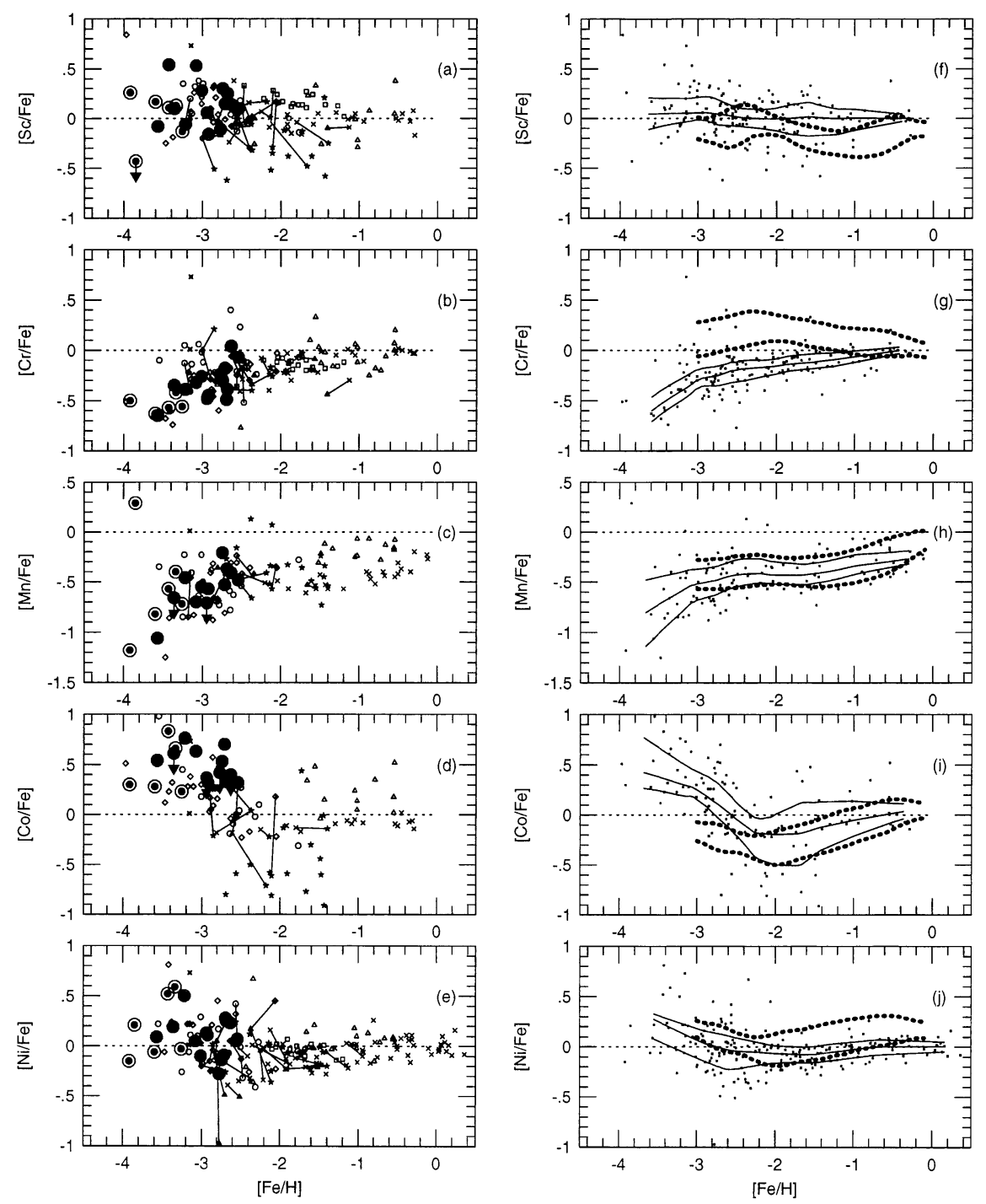

Figure 2. Relative abundances of the iron-peak elements as a function of $[\mathrm{Fe} / \mathrm{H}]$ from Ryan et al. (1996). The presentation is the same as described for Figure 1.

strates the discovery of McWilliam et al. (1995) that $\mathrm{Cr}$ and Mn, which lie below Fe in the Periodic Table, are underproduced relative to solar values at $[\mathrm{Fe} / \mathrm{H}]<-3.0$, while $\mathrm{Co}$, lying above $\mathrm{Fe}$, is overproduced. These effects are not predicted by standard supernova enrichment models, and provide an important challenge for future work. 


\subsection{THE HEAVY NEUTRON-CAPTURE ELEMENTS}

The observational results for the heavy neutron-capture elements show a much more complicated situation, as is shown in Figure 3. (No theoretical predictions of the type presented by Timmes et al. at lower atomic number are available for these elements.) Below $[\mathrm{Fe} / \mathrm{H}] \sim-2.5$ there is a large spread in these elements, which for $\mathrm{Sr}$ covers a range of a factor of 100 . The simplest explanation of these observations is that chemical enrichment at the earliest times was a somewhat patchy business.
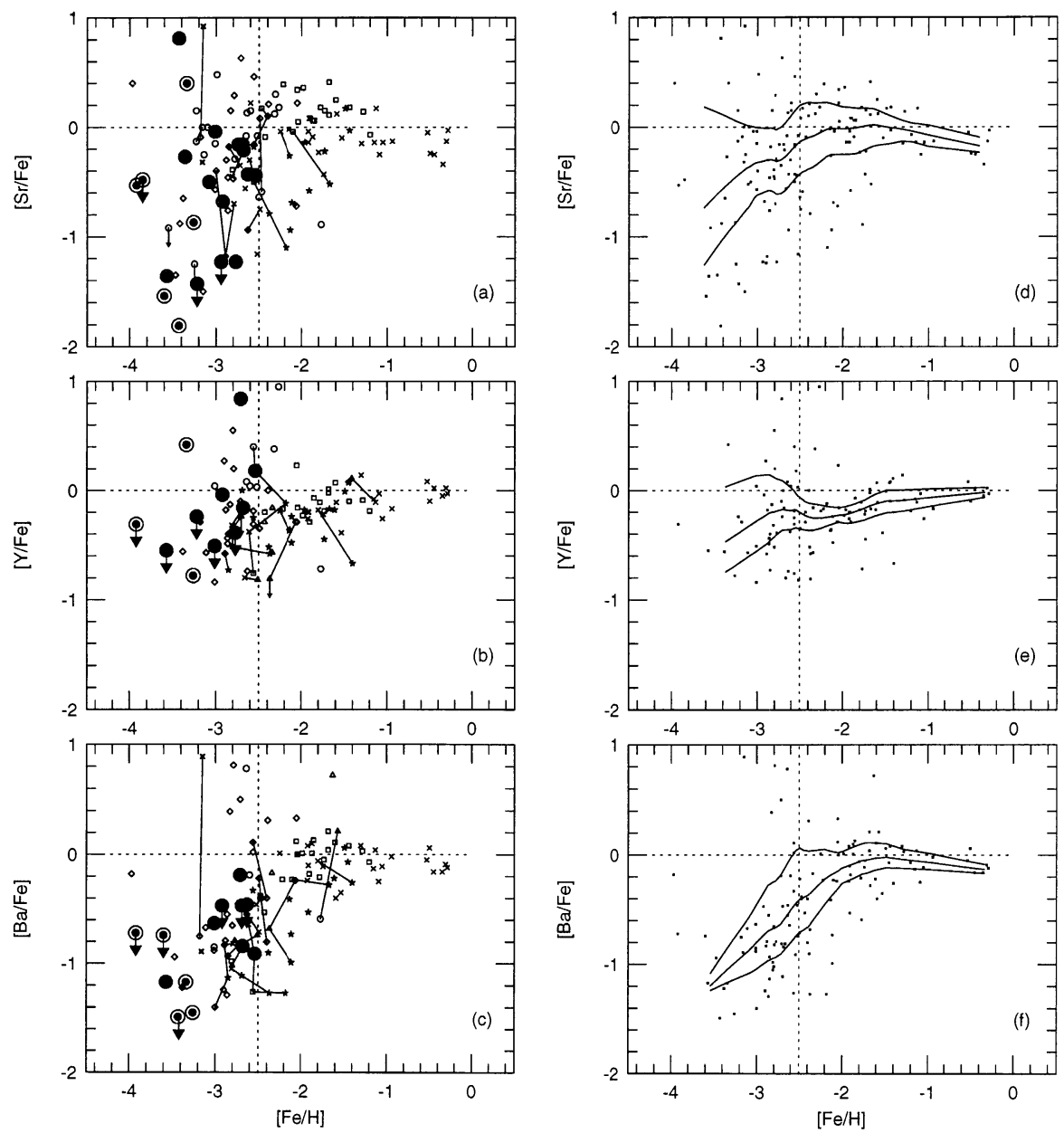

Figure 3. Relative abundances of the neutron-capture elements as a function of $[\mathrm{Fe} / \mathrm{H}]$ from Ryan et al. (1996). The presentation is similar to that described for Figure 1.

One of the most important aspects of the abundance patterns of the heavy elements is that while at high abundance stars such as the Ba II giants 
clearly possess the signature of the s-process and identify ${ }^{13} \mathrm{C}(\alpha, \mathrm{n}){ }^{16} \mathrm{O}$ as the neutron source (see e.g. Tomkin \& Lambert 1979, 1983), one finds at $[\mathrm{Fe} / \mathrm{H}]<-2.0$ the distribution is an r-process one (see e.g. McWilliam et al. 1995). The former receives its explanation in production of the heaviest elements in low- to intermediate-mass stars, while the latter presumably results from production in high-mass ones at the earliest times, perhaps in the formation of neutron stars as described by Woosley et al. (1994).

Of particular interest is the work of Sneden et al. $(1994,1996)$ on the metal-poor star CS 22892-052 $([\mathrm{Fe} / \mathrm{H}]=-3.0)$, which displays relative overabundances of the neutron-capture elements by $\sim 1 \mathrm{dex}$, in an r-process pattern. The overabundances are so large that the Th/Eu ratio may be determined, leading to an age estimate (and thus of the Galaxy) of $17 \pm$ $4 \mathrm{Gyr}$ (Cowan et al. 1996). Another important aspect is that it has [C/Fe] $=1.0$. As far as the author is aware none of the current concepts of stellar nucleosynthesis simultaneously produces both $\mathrm{C}$ - and r-process enrichment (see Norris et al. 1997 for further discussion of this point).

\section{Summary}

Theoretical calculations of stellar nucleosynthesis provide a basic understanding of most of the chemical abundance trends observed in stars. Important inadequacies, however, remain. Of these:

- Some are no doubt associated with problems inherent in the difficulty of modelling supernova explosions (e.g. absolute yields, $\mathrm{Cr}, \mathrm{Mn}, \mathrm{Co}$, and r-process heavy element production at low $[\mathrm{Fe} / \mathrm{H}])$.

- Others are suggestive of mixing phenomena (some driven perhaps by rotation?) not included or treated only approximately in the models (e.g. $\mathrm{N}$ at earliest times, $\mathrm{CNO}$, and $\mathrm{AlMgNa}$ in globular clusters).

- Some may indicate basic problems in the modelling (e.g. C-rich/ rprocess rich metal-poor stars such as CS 22892-052, Na variations on the main sequences of globular clusters, $\alpha$ elements in bulge giants).

- Some indicate a need for better model atmosphere abundance determination (e.g. non-LTE effects for $\mathrm{Al}$ in metal-weak stars).

- Some indicate a need for more and better observational data (e.g. $\alpha$ elements in bulge giants).

\section{References}

Arnett, D. 1996, Supernovae and Nucleosynthesis (Princeton : Princeton University Press)

Boesgaard, A. M. \& Friel, E. D. 1990, ApJ, 351, 467

Briley, M. M., Smith, V. V., Suntzeff, N. B., Lambert, D. L., Bell, R. A., \& Hesser, J. E. 1996, Nature, 383, 604

Burbidge, E. M., Burbidge, G. R., Fowler, W. A., \& Hoyle, F. 1957, Rev.Mod.Phys., 29, 547 
Busso, M., Picchio, G., Gallino, R., \& Chieffi, A. 1988, ApJ, 326, 196

Cameron, A. G. W. 1957, PASP, 69, 201

Couchman, H. M. P. \& Rees, M. J. 1986, MNRAS, 221, 53

Cowan, J. J., McWilliam, A., Sneden, C., \& Burris, D. L. 1996, preprint

Cowie, L. L., Songaila, A., Kim, T. -S., \& Hu, E. M. 1995, AJ, 109, 1522

Edvardsson, B., Andersen, J., Gustafsson, B., Lambert, D. L., Nissen, P. E., \& Tomkin, J. 1993, A\&A, 275, 101

Gibson, B. K. 1997, MNRAS, submitted

Hoyle, F. 1946, MNRAS, 106, 343

Iben, I. Jr. \& Truran, J. W. 1978, ApJ, 220, 980

Kajino, T., Mathews, G. J., \& Fuller, G. M. 1990, ApJ, 364, 7

King, J. 1994, AJ, 107, 350

Lambert, D. L. \& Ries, L. M. 1981, ApJ, 248, 228

McWilliam, A., Preston, G. W., Sneden, C., \& Searle, L. 1995, AJ, 109, 2757

McWilliam, A. \& Rich, R. M. 1994, ApJS, 91, 749

Nomoto, K., Thielemann, F. -K., \& Yokoi, K. 1984, ApJ, 286, 644

Norris, J. E., Ryan, S. G., \& Beers, T. C. 1997, ApJ, submitted

Pettini, M., Smith, L. J., Hunstead, R. W., \& King, D. L. 1994, ApJ, 426, 79

Renzini, A. \& Voli, M. 1981, A\&A, 94, 175

Ryan, S. G. \& Norris, J. E. 1991, AJ, 101, 1865

Ryan, S. G., Norris, J. E., \& Beers, T. C. 1996, ApJ, 471, 254

Sneden, C., McWilliam, A., Preston, G. W., Cowan, J. J., Burris, D. L., \& Armosky, B. J. 1996, ApJ, 467, 819

Sneden, C., Preston, G. W., McWilliam, A., \& Searle, L. 1994, ApJ, 431, L27

Tomkin, J. \& Lambert, D. L. 1979, ApJ, 227, 209

Tomkin, J. \& Lambert, D. L. 1983, ApJ, 273, 722

Timmes, F. X., Woosley, S. E., \& Weaver, T. A. 1995, ApJS, 98, 617

Trimble, V. 1975, Rev.Mod.Phys., 47, 877

Truran, J. W. 1984, ARN\&PS, 34, 53

Wagoner, R. V., Fowler, W. A., \& Hoyle, F. 1967, ApJ, 148, 3

Wheeler, J. C., Sneden, C., \& Truran, J. W. Jr. 1989, ARA\&A, 27, 279

Woosley, S. E. \& Weaver, T. A. 1982, in Supernovae: A Survey of Current Research, eds. M.J. Rees \& R.J. Stoneham, (Dordrecht : Reidel), 79

Woosley, S. E. \& Weaver, T. A. 1995, ApJS, 101, 181

Woosley, S. E., Wilson, J. R., Mathews, G. J., Hoffman, R. D., \& Meyer, B. S. 1994, ApJ, 433, 229

Zinn, R. 1985, ApJ, 293, 424

\section{Discussion}

S. Balachandran: I thought your $\mathrm{C}$ results at low metallicities very interesting. Can you tell me which $\mathrm{C}$ transition you used?

Norris: At lowest abundance only the $\mathrm{G}$ band is useful.

N. Langer: The scatter in $[\mathrm{r} / \mathrm{Fe}]$ you find at very low metallicity is really amazing. Can you disentangle whether mainly the scatter in the r-process abundances or in iron is responsible; i.e. if you would plot $[\mathrm{r} / \mathrm{O}]$ vs. $[\mathrm{O} / \mathrm{H}]$, and $[\mathrm{Fe} / \mathrm{O}]$ vs. $[\mathrm{O} / \mathrm{H}]$, which curve would have the strong scatter.

Norris: Unfortunately $\mathrm{O}$ abundances are not available at lowest abundance because of the weakness of the features. If, however, one were to use the $\alpha$ elements as proxy the scatter would be in the $[\mathrm{r} / \alpha]$ vs. $[\alpha / \mathrm{H}]$ plane rather than in the $[\mathrm{Fe} / \alpha]$ vs. $[\alpha / \mathrm{H}]$ one. 